\title{
Parameter Calibration Method of Microscopic Traffic Flow Simulation Models based on Orthogonal Genetic Algorithm
}

\author{
Yanfang Yang \\ School of Traffic and Transportation \\ Beijing Jiaotong University \\ Beijing, P. R. China \\ E-mail: yangyf@bjtu.edu.cn \\ Honghui Dong \\ School of Traffic and Transportation \\ Beijing Jiaotong University \\ Beijing, P. R. China \\ Email: hhdong@bjtu.edu.cn
}

\author{
Yong Qin \\ State Key Laboratory of Rail Traffic Control and Safety \\ Beijing Jiaotong University \\ Beijing, P. R. China \\ Corresponding author, Email: yqin@bjtu.edu.cn \\ Qing Zhang \\ Beijing Research Center of Urban Traffic Information \\ Sensing and Service Technologies \\ Beijing Jiaotong University \\ Beijing, P. R. China \\ Email: 15125808@bjtu.edu.cn
}

\begin{abstract}
Traffic microscopic traffic simulation models have become extensively used in both transportation operations and management analyses, which are very useful in reflecting the dynamic nature of transportation system in a stochastic manner. As far as the microscopic traffic flow simulation users are concerned, the one of the major concerns would be the appropriate calibration of the simulation models. In this paper a parameter calibration method of microscopic traffic flow simulation models based on orthogonal genetic algorithm is presented. In order to improve the capacity of locating a possible solution in solution space, the proposed method incorporates the orthogonal experimental design method into the genetic algorithm. The proposed method is applied to an arterial section of Ronghua Road in Beijing. Through comparing with the parameter calibration method based on genetic algorithm, the advantage of the proposed method is shown.
\end{abstract}

Keywords-Microscopic traffic flow simulation model; Parameter calibration; Orthogonal genetic algorithm; VISSIM

\section{INTRODUCTION}

Traffic simulation has become an important and popular tool in modeling transport system, with the progress of simulation technologies [1]. Traffic simulation models could be divided into three categories, including microscopic, macroscopic, mesoscopic simulation models. Microscopic simulation models simulate traffic at a level of individual vehicles [2]. Car-following and lane-changing models are the two fundamental components in microscopic simulation models. Macroscopic simulation models simulate transportation network section-by-section rather than tracking individual vehicles. Mesoscopic traffic simulation models combine the properties of the microscopic and macroscopic simulation models. For the traffic simulation models, the simulation results depend on the initial choice of the model [3] and the success of the calibration process [4].

The calibration of traffic microscopic simulation models is defined as the process of finding optimal parameters to match the field data so model will accurately represent field measure or observed traffic condition [5]. The optimization task involves comparing and minimization differences of selected indicators, e.g., travel time and queuing length [4], delays [6], travel time distribution [7], saturation flow rates [8] and emission [9], between the calibration model and the ones counted and measured in local traffic network.

Calibrating traffic model of bigger special and time scopes of a traffic network needs deal with a larger number of input parameters and calculating processes. In order to decrease time consumption, artificial intelligent techniques are applied into the calibration of traffic microscopic simulation model. Genetic algorithm (GA) has become the most common used calibration algorithm for input parameters of the simulations [5, 10-13], since Cheu et al., firstly used GA calibrating FRESIM model [14]. Other intelligent algorithms are also used in the calibration of traffic simulation, e.g., perturbation stochastic approximation (SPSA) scheme [15], particle swarm optimization (PSO) [16], and neural network approach [4]. These methods automate the calibration process to a certain degree and it was generally reported that they improve simulation performance over the default model parameter values.

Microscopic simulation is a complex system that all parameters work together to influence its modeling results. In calibrating such a complex model, users could get trapped in 
the local optima of the objective function, due to the high dimension and numerous local optima. This paper focuses on the above question of GA when calibrating the driving behavior model parameters in VISSIM.

The exposition of this paper is as follows: the next section implements the orthogonal genetic algorithm (OGA). Some studies have found that applying an experimental design method (orthogonal design) into GA may overcome the limitation mentioned above [17-18]. The third section gives the procedure of calibrating the microscopic traffic flow simulation model based on OGA. The fourth section applies OGA calibration method to the calibration of a signal intersection in Beijing, and comparing with the GA and orthogonal design method respectively. The final section summarizes the paper.

\section{CALIBRATION METHOD BASED ON OGA}

The proposed calibration method employs an orthogonal genetic algorithm. This section contains a brief overview of the VISSIM calibration parameters set, and the fundamentals of the OGA, including the structure of chromosome, fitness function and orthogonal crossover decoding.

\section{A. Selection of Parameters}

In this paper, a microscopic traffic flow simulation, VISSIM, is selected as the basic platform for the parameter calibration. VISSIM models the psychophysical driver behavior and attempts to capture both the physical and the human components of traffic [19]. Parameters of two driving behavior models are in considered in this paper: the carfollowing model and the lane-changing model. After parameter sensitivity analysis, i.e., one-way Analysis of Variance (ANOVA)[20], four parameters are selected as the calibration parameters. Table 1 lists the calibration parameter set, including default value, the minimum and maximum value.

\section{TABLE 1 CALIBRATION PARAMETER SET}

\begin{tabular}{lcccc}
\hline Parameters $\left(\boldsymbol{x}_{\mathbf{j}}\right)$ & Unit & $\begin{array}{c}\text { Default } \\
\text { Value }\end{array}$ & $\begin{array}{c}\text { Min } \\
\left(\boldsymbol{u}_{j}\right)\end{array}$ & $\begin{array}{c}\text { Max } \\
\left(\boldsymbol{v}_{j}\right)\end{array}$ \\
\hline $\begin{array}{c}x_{1} \text { Average standstill } \\
\text { distance }\end{array}$ & $\mathrm{m}$ & 2 & 0.5 & 3 \\
$\begin{array}{c}x_{2} \text { Additive part of desired } \\
\text { safety distance }\end{array}$ & $\mathrm{NA}$ & 2 & 0.5 & 3 \\
$\begin{array}{c}x_{3} \text { Multiple part of desired } \\
\text { safety distance }\end{array}$ & $\mathrm{NA}$ & 3 & 1 & 6 \\
$x_{4}$ Maximum deceleration & $\mathrm{m} / \mathrm{s}^{2}$ & 4 & 2 & 6 \\
\hline
\end{tabular}

\section{B. The Structure of Chromosomes}

Supposing $P_{i}\left(x_{i 1}, \ldots, x_{i N}\right)$ is the $i$ th chromosome, $x_{i j}$ is the $j$ th parameter value in the $i$ th chromosome. $\forall i=1,2, \ldots, M$, $j=1,2, \ldots, N=4 . M$ is the total number of chromosomes and $N$ is the number of parameters to be calibrated. $[\boldsymbol{l}, \boldsymbol{u}]=\left[\left(l_{1}, \ldots, l_{N}\right)\right.$, $\left.\left(u_{1}, \ldots, u_{N}\right)\right]$ defines the feasible solution space and the corresponding domain of $x_{j}$ is $\left[l_{j}, u_{j}\right]$ (e.g., the domain of $x_{1}$ is $[0.5,3]$ as listed in Table 1$)$. In this paper, parameters to be calibrated are coded into chromosomes, which quantized by orthogonal design. We quantize the domain $\left[l_{j}, u_{j}\right]$ of $x_{j}$ into $Q$ levels, where the design parameter $Q$ is odd.

Algorithm 1 shows the procedure of constructing chromosome. Firstly, we calculate $M$, where $M=Q^{J}, J$ is the smallest positive integer fulfilling $J \geq \frac{\log (N(Q-1)+1)}{\log Q}$. Secondly, we construct the orthogonal array $L_{M}\left(Q^{N}\right)$ corresponding to the chromosome. Each element $a_{i j}$ of the orthogonal array $L_{M}\left(Q^{N}\right)$ represents the levels numbers in orthogonal design, $a_{i j} \in\{0,1, \ldots, Q-1\}, \forall i=1,2, \ldots, M, j=1,2, \ldots$, $N$. Finally, the corresponding parameter value $x_{i j}$ of $a_{i j}$ in feasible solution pace $\left[l_{j}, u_{j}\right]$ is calculated by the equation as shown in follows:

$$
x_{i j}=l_{j}+a_{i j} \times \frac{u_{j}-l_{j}}{Q-1}, \quad \forall 0 \leq a_{i j} \leq Q-1
$$

\section{Algorithm 1: Constructing chromosomes}

Step 1. Calculating the number of chromosome, $M=Q^{J} . J$ is the smallest positive integer fulfilling $J \geq \frac{\log (N(Q-1)+1)}{\log Q}$.

Step 2. Construct the orthogonal array $L_{M}\left(Q^{N}\right)$

(1) Construct the basic columns:

$$
\begin{aligned}
& \text { for } k=1 \text { to } J \text { do } \\
& j=\frac{Q^{k-1}-1}{Q-1}+1 \\
& \text { for } i=1 \text { to } Q^{J} \text { do } \\
& \quad a_{i j}=\left\lfloor\frac{i-1}{Q^{J-1}}\right\rfloor \bmod Q \\
& \text { end for } \\
& \text { end for }
\end{aligned}
$$

(2) Construct the non-basic columns:

$$
\text { for } k=2 \text { to } J \text { do }
$$

$$
\begin{aligned}
& j=\frac{Q^{k-1}-1}{Q-1}+1 \\
& \text { for } s=1 \text { to } j-1 \text { do } \\
& \text { for } t=1 \text { to } Q-1 \text { do } \\
& a_{j+(s-1)(q-1)+t}=\left(a_{s} \times t+a_{j}\right) \bmod Q \\
& \text { end for }
\end{aligned}
$$

(3) Selecting the first $N$ columns to construct the chromosome encoding array $L_{M}\left(Q^{N}\right)$

Step 3. Calculate $x_{i j}$ to construct chromosome using (1).

\section{Fitness Function}

The fitness function is a combination of the root mean absolute square error (RMASE) of travel time (TT) and maximum queue length (MQL) and between the VISSIM output and field data. The fitness function takes the form of 


$$
\begin{gathered}
F_{\text {total }}=\frac{1}{\xi \cdot E_{1}+(1-\xi) E_{2}} \\
E_{n}=\sqrt{\frac{1}{T} \sum_{t}\left(\frac{d_{n t}^{s}-d_{n t}^{o}}{d_{n t}^{o}}\right)^{2}} \forall n=1,2, t=1,2, \ldots, T
\end{gathered}
$$

where

$E_{1}=$ the RMASE of TT

$E_{2}=$ the RMASE of MQL

$T=$ the number of detector station;

$d_{n t}^{s}=$ the simulation model output of $t$ th sensor

$d_{n t}^{o}=$ the field data of $t$ th sensor station.

\section{Adaptive orthogonal crossover operator}

For each pair of parents (denoted by $P_{1}\left(x_{11}, x_{12}, x_{13}, x_{14}\right)$ and $P_{2}\left(x_{21}, x_{22}, x_{23}, x_{24}\right)$ ), performing the adaptive orthogonal crossover operation with the probability of crossover $p_{c}$. Adaptive orthogonal crossover algorithm is shown in Algorithm 2. $\sigma_{0}$ is the similarity threshold of each dimension between two parents .

Algorithm 2: Constructing chromosomes

Step 1. Calculating the number of similar dimension using $b=\operatorname{Num}\left(\left|x_{1, j}-x_{2, j}\right|>\sigma_{0}\right)$

Step 2. Constructing orthogonal array $L_{E}\left(F^{b}\right)=\left[a_{t s}\right]_{E \times b}$, with $b$ factors and $F$ levels, using Algorithm 1.

Step 3. Generating $E$ chromosomes as the potential offspring, $P_{t}^{\prime}\left(x_{t, 1}^{\prime}, x_{t, 2}^{\prime}, x_{t, 3}^{\prime}, x_{t, 4}^{\prime}\right), \forall t=1,2, \ldots, E$.

$$
\begin{aligned}
& \text { for } t=1 \text { to } E \text { do } \\
& s=1 \\
& \text { for } j=1 \text { to } 4 \text { do } \\
& \text { if }\left|x_{1 j}-x_{2 j}\right| \leq \sigma_{0} \\
& x_{t j}^{\prime}=\frac{x_{1 j}+x_{2 j}}{2} \\
& \text { else if }\left|x_{1 j}-x_{2 j}\right|>\sigma_{0} \\
& x_{t j}^{\prime}=\min \left(x_{1 i}, x_{2 i}\right)+a_{t s} \frac{\max \left(x_{1 i}, x_{2 i}\right)-\min \left(x_{1 i}, x_{2 i}\right)}{F-1} \\
& s=s+1 \\
& \text { end if } \\
& \text { end for } \\
& \text { end for }
\end{aligned}
$$

Step 4. Calculating the fitness values corresponding to each potential offspring and selecting the chromosome corresponding to the max fitness value as the offspring of two parents $P_{1}$ and $P_{2}$.

In this paper, $\sigma_{0}=0.005$ and $F=2$.

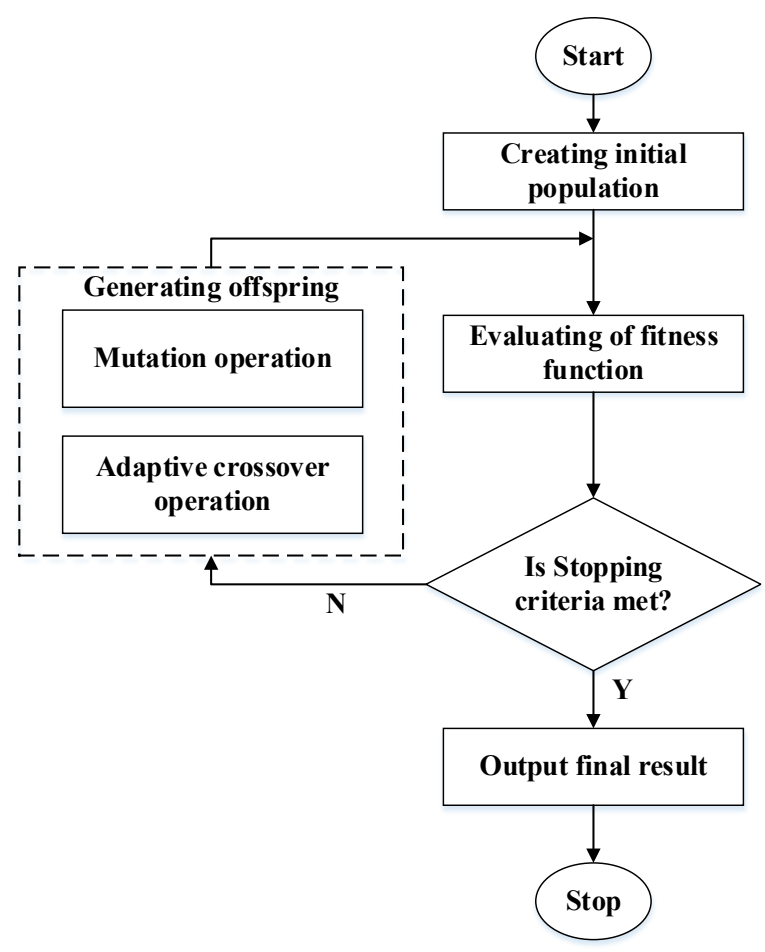

Figure 1 Calibration procedure

III. CALIBRATION PROCEDURE

We develop the parameter calibration program using VB language. The details of overall algorithm are as follows (see Fig. 1).

Step 1: Initialization

Executing Algorithm 1 to generate $M$ potential parents (e.g., levels $Q=9$ and $M=Q^{J}=9^{2}=81$ ). The microscopic simulation traffic simulation model VISSIM is run with the $M$ parameter group as the input file successively. Then $M$ fitness values corresponding to the potential parents are calculated. Sort the potential parents in a descending sort order, according the fitness values. Select the first $I:=50$ parents as the potential initial population $P_{0}$. In order to keep the individual distributing uniformly, we select $D:=10$ chromosomes randomly from the $D$ potential initial population as the initial population, denoted by $P_{\text {gen }=1}$.

Step 2: Adaptive crossover operation

For an arbitrary pair of parents, adaptive crossover operation is performed with the probability of crossover $P_{c}$ (e.g., $P_{c}=0.75$ ) using algorithm in Algorithm 2 and generate offspring population $C_{g e n}$.

Step 3: Mutation operation

Each chromosome in $P_{\text {gen }}$ would undergo mutation operation with the probability of mutation $p_{m}$ (e.g., $p_{m}=0.1$ ). The mutation operation is as follows: (1) randomly generating an integer $j \in[1, N]$ and a real number $z \in\left[l_{j}, u_{j}\right]$; (2) replacing 
the $j$ th component of the chosen chromosome by $z$ to get a new chromosome. The mutation operation generates a new population denoted by $G_{g e n}$. The fitness values corresponding to each new chromosome in $G_{g e n}$ are calculated by running VISSIM model.

Step 4: Selection operation

In order to maintain the population diversity, we sort the population $\left(P_{\text {gen }}+C_{\text {gen }}+G_{\text {gen }}\right)$ in a descending sort order according the fitness values, then select the first $\lfloor D * 70 \%\rfloor$

chromosomes and randomly select $D-\lfloor D * 70 \%\rfloor$ chromosomes from the rest of $\left(P_{g e n}+C_{g e n}+G_{g e n}\right)$ to constructing the next population $P_{\text {gen }+1}$.

Step 5: Check stopping rules

Supposing the max fitness values of iteration gen is $F_{\max }^{g e n}$. If gen $=$ the maximum number of iterations or $\left|F_{\max }^{\text {gen }}-F_{\max }^{\text {gen }-1}\right| \leq 0.005$, the program stops. Otherwise, go to Step 2 and gen $=$ gen +1 .

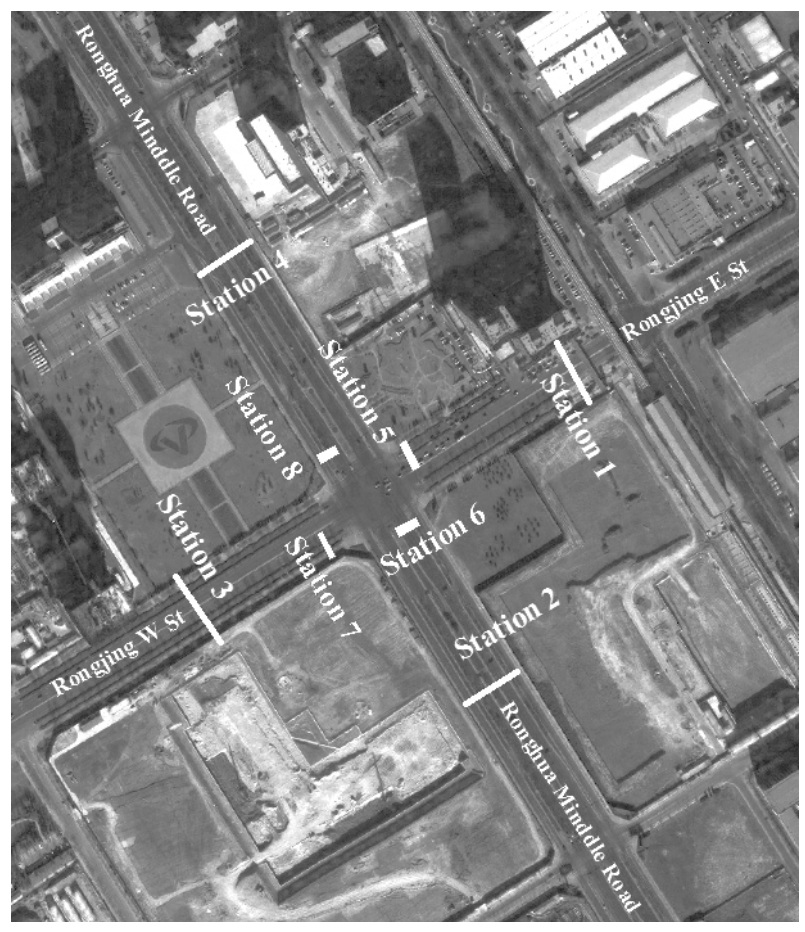

Figure 2 The location distribution of detector stations

\section{CASE STUDY}

A signal intersection constructed by the Ronghua middleroad and the Rongjing road in Beijing is selected as the test bed. The intersection locates at the arterial section of Beijing economic-technological development area. The location and map are shown in Fig. 2. Field data were gathered at evening peak period (18:15-19:15) in July 25, 2011. The traffic volumes were video-taped by four cameras, which were located at the Station 5-Station 8 in Fig. 2, respectively. We recorded the maximum queue length at the entrance (i.e., Station 5Station 8 in Fig. 2) into the intersection every signal cycle and use the mean of the above queue length as the hourly maximum queue length. The travel time was collected by floating cars. Fig. 2 shows the start and end points of travel time collection detectors, e.g., the Northwest-bound travel time collection is from Station 2 to Station 4, the Southeast-bound travel time collection is from Station 4 to Station 2.

The traffic model used is VISSIM Version 5.30. After building the VISSIM model, we apply the OGA to the parameter calibration. The default size of initial population $D$ is 10 . When the difference of maximum fitness values of two consecutive iterations is no more than 0.005 or the maximum number of iterations is 50 , the program stops. Table 2 lists the relative errors of traffic volumes between VISSIM output results and field data at four entrances respectively, which shows that the values are all no more than $2.21 \%$.

TABLE 2 VOLUME CALIBRATION OF THE INVESTIGATED INTERSECTION

\begin{tabular}{cccc}
\hline & $\begin{array}{c}\text { Simulation } \\
\text { Results } \\
(\text { vehicle/h) }\end{array}$ & $\begin{array}{c}\text { Field Data } \\
\text { (vehicle/h) }\end{array}$ & $\begin{array}{c}\text { Relative } \\
\text { Error }\end{array}$ \\
\hline Northeast-bound & 1173 & 1170 & $0.26 \%$ \\
Southwest-bound & 486 & 497 & $2.21 \%$ \\
Southeast-bound & 1766 & 1782 & $0.9 \%$ \\
Northwest-bound & 525 & 528 & $0.57 \%$ \\
\hline
\end{tabular}

The proposed method is compared with calibration with the GA method and the orthogonal design method. In GAbased parameter calibration experiments, the fitness function, the size of initial population and the stopping rules are consistent with the OGA-based parameter calibration experiments. In the orthogonal design method, level number $Q$ is 9 , and the weight coefficient is 0.5 . In the orthogonal design experiments, the parameter set is quantized by orthogonal design. The level number $Q$ is the same with the OGA and the GA. The orthogonal array is constructed using Algorithm 1. Using the $Q^{J}=81$ parameter groups as the VISSIM input data and selecting the maximum fitness value as the final output results.

We compare the maximum fitness values corresponding to four methods (i.e., default value, OGA, GA and the orthogonal method). The RMASE of travel time, RMASE of maximum queue length and fitness values are listed in Table 3. The maximum fitness value of OGA is 19.43 , which is much bigger than that of other methods. 
TABLE 3 COMPARATIVE ANALYSIS OF THE FITNESS FUNCTION VALUE

\begin{tabular}{llllll}
\hline Methods & $\begin{array}{l}\text { Parameter } \\
\text { values }\end{array}$ & $\begin{array}{l}\text { TT } \\
\text { RMASE }\end{array}$ & $\begin{array}{l}\text { MQL } \\
\text { RMASE }\end{array}$ & Weight & Fitness \\
\hline Default & {$[2,2,3,-4]$} & 0.0607 & 0.3372 & 0.5 & 5.03 \\
OGA & $\begin{array}{l}{[1.125,0.5,} \\
2.25,-2.75]\end{array}$ & 0.0225 & 0.0805 & 0.5 & 19.43 \\
GA & $\begin{array}{l}{[1.2,2.6,} \\
1.33,-6]\end{array}$ & 0.0287 & 0.1380 & 0.5 & 11.99 \\
$\begin{array}{l}\text { Orthogonal } \\
\text { method }\end{array}$ & {$[0.5,0.813$,} & 0.0430 & 0.1378 & 0.5 & 11.06 \\
\hline
\end{tabular}

Calculate the average errors for maximum queue length and travel time, respectively. The formula is as follows:

$$
\varepsilon_{i}=0.25 \sum_{i=1}^{4} \frac{\left|d_{n t}^{s}-d_{n t}^{0}\right|}{d_{n t}^{0}} \quad n \in\{1,2\}
$$

where,

$\varepsilon_{1}=$ the average errors of TT

$\varepsilon_{2}=$ the average errors of MQL

$d_{n t}^{s}=$ simulation output of $t$ th entrance

$d_{n t}^{0}=$ field data of $t$ th entrance

$t=1,2,3,4$ represent four entrance, i.e., northeast-bound, southwest-bound, southeast-bound and northwest-bound.

Fig. 3 and Fig. 4 represent the relative error of each access respectively. The relative error of the proposed method is less than that of other methods. Meanwhile, the relative error of travel time is higher than that of queue length, because investigators collect the queue length according to estimating the length of vehicles. It is obviously that the error must be existed.

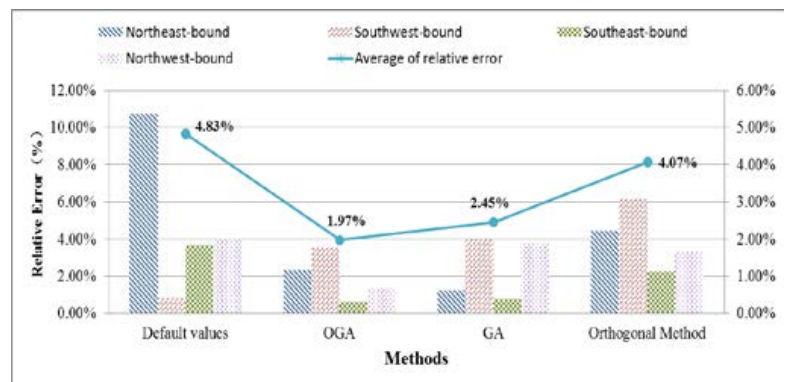

Figure 3 Comparison of TT relative error

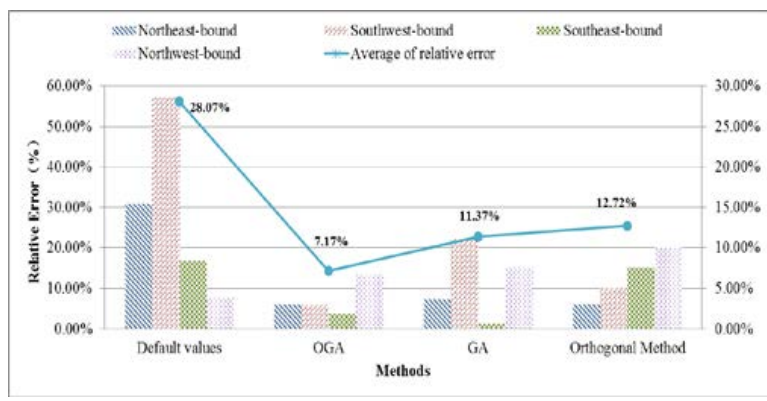

Figure 4 Comparison of MQL relative error

Fig.5 shows the comparison of convergence performance between the OGA-base calibration method and the GA-based calibration method. The former method stops when the iteration count is 10 . Otherwise, the GA-based calibration method stops when iteration count is 40 . To the OGA-base calibration method, it is obviously that constructing initial population consumes the most part of consuming time, because the program needs to run VISSIM $9^{2}=81$ times to generate the output results. So we count the number of running VISSIM. Throughout the procedure, the proposed method runs VISSIM 238 times, and the later method runs 400 times.

Considering how the weight coefficient value impacts the calibration results. Fig. 6 shows the profile of the fitness values versus weight coefficient values (i.e., weight coefficient value $=0,0.2,0.5,0.8$, and 1.0, respectively). The figure shows that a significant correlation exists between the fitness value and the weight coefficient values. The bigger the weight coefficient value is, the bigger the fitness value is. Considering the case of weight coefficient $=1.0$, which means taking travel time into account indicator only, the fitness value is 62.70. Maximum queue length may reduce the fitness value, because field data collection exist big error.

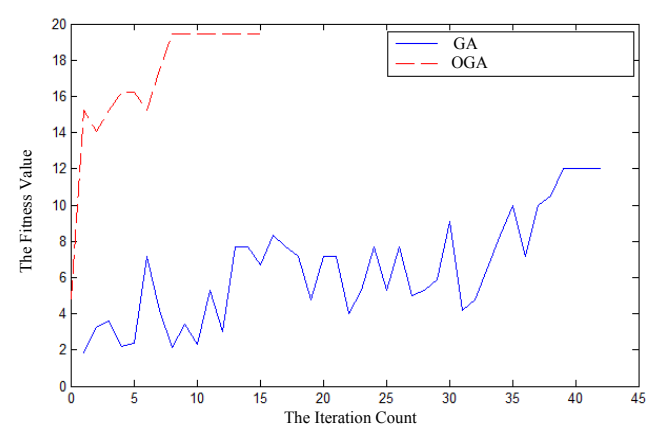

Figure 5 Comparison of convergence rate between two algorithms 


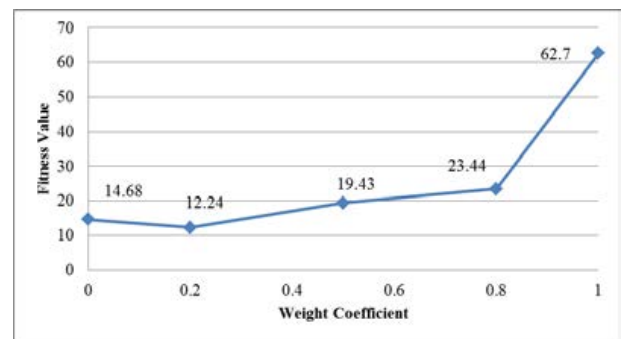

Figure 6 Comparison of the fitness value versus weight coefficient

\section{CONCLUSION}

This paper focuses on the automatic calibration method of traffic micro-simulation. A parameter calibration method based on orthogonal genetic algorithm is proposed. The first step, the process and the pseudo code of the OGA-based microsimulation calibration method are given. The second step, we apply the proposed method to a signal intersection in Beijing. In the case study, the microscopic traffic flow simulation model VISSIM is selected. We compare the OGA method with the GA method and the orthogonal design method, respectively, i.e., the maximum fitness value, the relative errors of TT and MQL, the number of iteration. Experiment results show that the OGA outperforms the GA and the orthogonal design method in calibration. This paper also analyzes how the weight coefficient impacts the calibration results. A significant correlation exists between the fitness value and the coefficient values.

\section{ACKNOWLEDGMENT}

This research was supported by the National Science \& Technology Support Program of China through Grant No. 2014BAG01B04.

\section{REFERENCES}

[1] D. Sun, L. Zhang, and F. Chen. "Comparative study on simulation performances of CORSIM and VISSIM for urban street network". Simulation Modeling Practice and Theory, Vol.37, pp. 18-29, 2013

[2] H. Filmon, and P. Luis Guilherme. "Sensitivity analysis of VISSIM driver behavior parameters on safety of simulated vehicle and their interaction with operations of simulated traffic". Transportation Research Board 92nd Annual Meeting, Washington DC, 2013

[3] F.C. Fang, and L. Elefteriadou. "Some guidenlines for selecting microsimulation models for interchange traffic operational analysis." Journal of Transportation Engineering, Vol. 131, No. 7, pp. 535-543, 2005
[4] I. I. Otković, T. Tollazzi, and M. Šraml. "Calibration of microsimulation traffic model using neural network approach. Expert Systems with Applications", Vol. 40, pp. 5965-5974, 2013

[5] B. Park, and J.D. Schneeberge. "Microscopic simulation modeling calibration and validation: a case study of VISSIM for a coordinated actual signal system". Transportation Research Record: Journal of the Transportation Research Board, pp. 185-192, 2003

[6] C. Gagnon, A.W. Sadek, and A. Touchette. "Calibration potential of common analytical and microsimulation roundabout models: new England case study". Transportation Research Record: Journal of the Transportation Research Board, pp. 77-86, 2008

[7] S. Kim, W. Kim, and L.R. Rilett. "Calibration of microsimulation models using nonparametric statistical techniques". Transportation Research Record: Journal of the Transportation Research Board, pp. 111-119, 2005

[8] J. Asamer, H. J.V. Zuylen, and B. Heilmann. "Calibrating car-following parameters for snowy road conditions in the microscopic traffic simulator VISSIM". IET Intelligent Transport System. Vol. 7, pp. 114121,2011

[9] L. Jie, H. V. Zuylen, Y. Chen, F. Viti, and I. Wilmink. "Calibration of a microscopic simulation model for emission calculation". Transportation Research Part C, Vol. 31, pp. 172-184, 2013

[10] B. Park, and H. Qi, "Development and evaluation of a procedure for the calibration of simulation models". Transportation Research Record: Journal of the Transportation Research Board, pp.208-217, 2005

[11] T. Ma, and B. Abdulhai. "Genetic algorithm-based optimization approach and genetic tool for calibrating traffic microscopic simulation parameters". Transportation Research Record 1806, pp. 6-15, 2002

[12] L.O. Kim, and L.R. Rilett. "A genetic algorithm based approach to traffic micro-simulation calibration using ITS data". Transportation Research Board 83rd Annual Meeting, Washington, D. C. 2004

[13] S. Menneni, C. Sun, and P. Vortisch. "Microsimulation calibration using speed-flow relationships". Transportation Research Record: Journal of the Transportation Research Board, pp.1-9, 2009

[14] R. L. Cheu, X. Jin, K.C. Ng, Y.L. Ng, and D. Srinivasan. "Calibration of FRESIM for Singapore expressway using genetic algorithm”. Journal of Transportation Engineering, Vol. 124, pp. 526-535, 1998

[15] J. Ma, D. Hu, and H. Dong, and H. Zhang, "Calibration of microsimulation with heuristic optimization methods". Transportation Research Record: Journal of the Transportation Research Board, pp. 208-217, 2007

[16] K. Aghabayk, M. Sarvi, W. Young, and L. Kautzsch. "A novel methodology for evolutionary calibration of VISSIM by multithreading". 36th Australasian Transport Research Forum, Australia, 2013

[17] Y. Wang, H. Liu, Z, Cai, and Y, Zhou. "An orthogonal design based constrain evolutionary optimization algorithm. Engineering Optimization”. vol.39,pp.715-736, 2007

[18] Y. Leung, and Y. Wang. "An orthogonal genetic algorithm with quantization for global numerical optimization". IEEE Transaction On Evolutionary Computation, vol.5,pp.41-52, 2011

[19] Brackstone, M., and M. McDonald. "Car-following: a historical review". Transportation Research Part F, pp.181-196, 1999

[20] Manijunatha, P., P. Vortisch, P, and T. V. Mathew. "Methodology for the Calibration of VISSIM in Mixed Traffic", Transportation Research Board 92nd Annual Meeting, Washington D. C., 2013 